Please do not remove this page

RMIT

UNIVERSITY

\title{
Insulation properties of the monolithic and flexible aerogels prepared at ambient pressure
}

Cai, Jackie; Lucas, Stuart; Wang, Lijing; Cao, Yi

https://researchrepository.rmit.edu.au/esploro/outputs/9921858307301341/filesAndLinks?institution=61RMIT_INST\&index=null

Cai, J., Lucas, S., Wang, L., \& Cao, Y. (2012). Insulation properties of the monolithic and flexible aerogels prepared at ambient pressure. Advanced Materials Research, 391-392(2012), 116-120.

https://doi.org/10.4028/www.scientific.net/AMR.391-392.116

Document Version: Accepted Manuscript

Published Version: https://doi.org/10.4028/www.scientific.net/AMR.391-392.116

Repository homepage: https://researchrepository.rmit.edu.au

(C) 2012 Trans Tech Publications

Downloaded On 2023/04/26 22:53:46 +1000

Please do not remove this page 
Thank you for downloading this document from the RMIT Research Repository.

The RMIT Research Repository is an open access database showcasing the research outputs of RMIT University researchers.

RMIT Research Repository: http://researchbank.rmit.edu.au/

\section{Citation:}

Cai, J, Lucas, S, Wang, L and Cao, Y 2012, 'Insulation properties of the monolithic and flexible aerogels prepared at ambient pressure', Advanced Materials Research, vol. 391392, no. 2012, pp. 116-120.

See this record in the RMIT Research Repository at: http://researchbank.rmit.edu.au/view/rmit:15790

Version: Accepted Manuscript

Copyright Statement: (c) 2012 Trans Tech Publications

Link to Published Version:

http://dx.doi.org/10.4028/www.scientific.net/AMR.391-392.116 


\title{
Insulation Properties of the Monolithic and Flexible Aerogels Prepared at Ambient Pressure
}

\author{
Jackie Y. Cai ${ }^{1, a, ~}{ }^{*}$, Stuart Lucas ${ }^{1, b}$, Lijing Wang ${ }^{2, c}$, Yi Cao ${ }^{1}$ \\ ${ }^{1}$ CSIRO Materials Science and Engineering, PO Box 21, Belmont, Victoria, 3216, Australia \\ ${ }^{2}$ School of Fashion and Textiles, RMIT University, 25 Dawson Street, Brunswick, Victoria 3056, \\ Australia \\ a email: Jackie.cai@csiro.au, ${ }^{\text {b email: Stuart.Lucas@csiro.au, }}{ }^{\text {c email: Lijing.Wang@rmit.edu.au }}$
}

Keywords: flexible aerogel, methyltrimethoxysilane, vinyltrimethoxysilane, thermal insulation, acoustic insulation.

\begin{abstract}
This paper reports a method for scale-up fabrication of hydrophobic and flexible aerogels derived from a combined precursor methyltrimethoxysilane and vinyltrimethoxysilane. The method developed enables the preparation of large pieces of monolithic aerogels at ambient pressure drying conditions. The thermal and acoustic insulation properties of the flexible aerogels were investigated and compared with the commercially available insulation materials.
\end{abstract}

\section{Introduction}

Aerogels are highly porous materials with lots of extreme properties. They are regarded as the materials of the 21st century, and potentially one of the most useful substances ever created [1]. However, to date their widespread applications have been limited due to their brittle and fragile nature and high manufacturing cost. In recent years, there has been a renewed interest in aerogel research due to the changing and challenging needs of the 21st century. In the face of an environmental and energy crisis, there are great needs for materials like aerogels that can conserve energy, and reduce greenhouse gas emissions and pollution (for instance, thermal and acoustic insulation, air and water purification). Because of this, intensive research interests have been making significant progress in aerogel's technology development and commercial applications. The global market for aerogels is growing rapidly, being driven primarily by insulation applications at present [2]. To fully realise the potential of aerogels, continued efforts are required to tackle the outstanding technical issues and improve the practical feasibility of the aerogel fabrication.

Recently, we have developed several processes for preparing monolithic areogels with low bulk densities, at ambient pressure without the need for any solvent exchange and surface modification [3-6]. This has been achieved through the use of the specified precursors methyltrimethoxysilane or a combination of methyltrimethoxysilane (MTMS) and vinyltrimethoxysilane (VTMS), and specifically tailored processing conditions. These precursors used have largely determined the flexible and hydrophobic characteristics of the resultant aerogels [3-7].

In this paper, we report a further modified process for scale-up preparation of the crack-free aerogels at ambient pressure based on the use of a combined precursor of MTMS-VTMS, and investigate the thermal and acoustic insulation properties of the aerogels prepared.

\section{Experimental Methods}

The MTMS-VTMS derived aerogels were prepared via a two-step, acid-base catalyzed sol-gel process, according to the optimised process described in [6] with slight modifications. In a typical experiment, a mixture of MTMS and VTMS $(\approx 4: 1)$ was mixed with methanol $(\mathrm{MeOH})$ at

\footnotetext{
* Corresponding author
} 
$\mathrm{MeOH} /$ precursors molar ratio of $24: 1$. The $0.01 \mathrm{M}$ aqueous solution of oxalic acid (Solution A) was added to the reaction media. The mixture was stirred at room temperature for 24 hours to complete the hydrolysis process. The 10M aqueous solution of ammonium hydroxide (Solution B) was then added dropwise to the as-prepared sol, in conjunction with a small proportion of trimethylamine (TMA) while stirring. The molar ratios of MTMS-VTMS/Solution A/Solution B were kept constant at 1:4:4. The prepared alcosol was transferred into a mold, which was then made air-tight and kept at $40^{\circ} \mathrm{C}$ for the gelation to occur. The alcogels were aged in $\mathrm{MeOH}$ for $24-48$ hours at $60^{\circ} \mathrm{C}$, then dried under ambient pressure at $50-60^{\circ} \mathrm{C}$ for 24 hours, then at $100^{\circ} \mathrm{C}$ for $2-3$ hours and finally at $150^{\circ} \mathrm{C}$ for 1 hour.

The large pieces of aerogels prepared were evaluated for their thermal and acoustic insulation properties. The thermal resistance $(\mathrm{R})$ of a sample was measured using KES-F Thermolabo II, as shown in Fig. 1. The thermal conductivity was calculated using the following equation:

Thermal Conductivity $\mathrm{k}\left(\mathrm{W} / \mathrm{m} .{ }^{\circ} \mathrm{C}\right)=\mathrm{D} / \mathrm{R}$

Where D is the thickness of the sample in metres. The sample thickness was measured using Shirley Thickness Gauge.

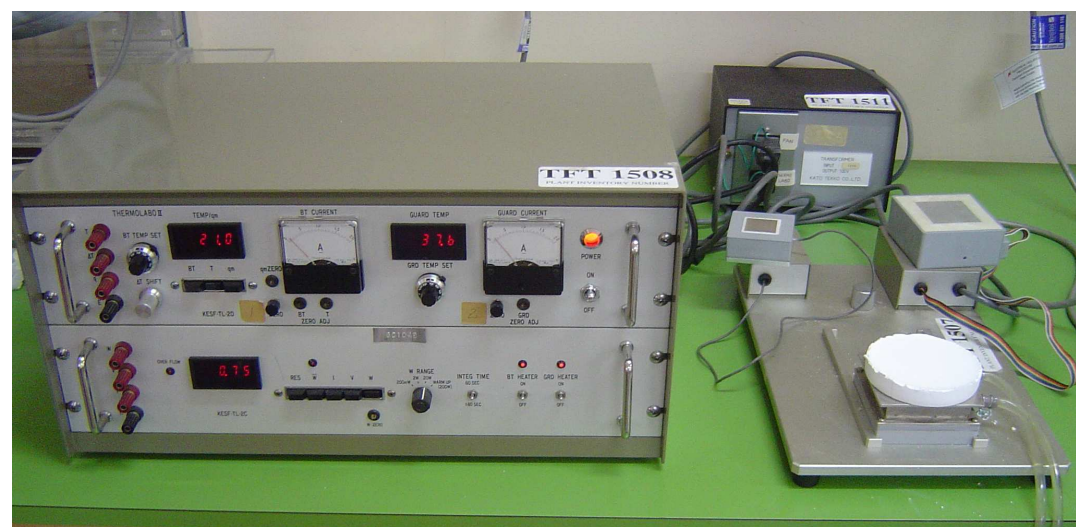

Figure 1. KES-F Thermolabo II

The acoustic absorption coefficient of a sample was measured using a Sound Impedance Tube, as illustrated in Fig. 2, according to the ASTM standard E1050-98 "Standard Test Method for Impedance and Absorption of Acoustical Materials Using A Tube, Two Microphones and A Digital Frequency Analysis System".

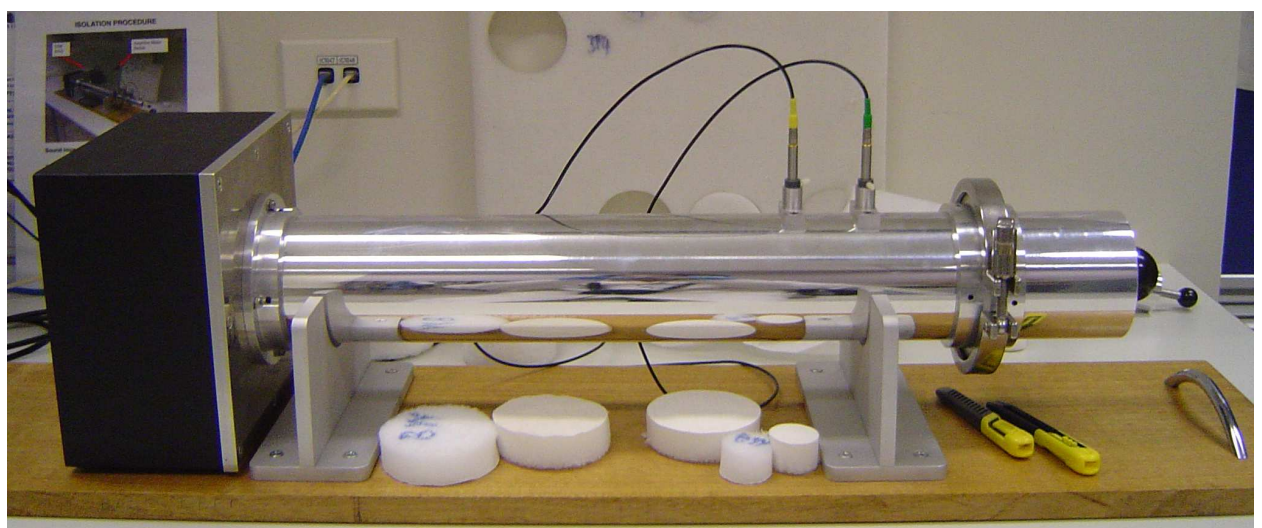

Figure 2. A Sound Impedance Tube 


\section{Results and Discussion}

Scale-up Preparation and Morphology of the Aerogels. Our previous investigation on MTMSVTMS based aerogel was carried out in a small scale, and the method developed was able to produce monolithic crack-free aerogels at ambient pressure with excellent reproducibility in test tubes. However, when the same process was employed for a scale-up production of the aerogel with a significantly different geometry (e.g. a wide and thin object), the crack-free aerogel was rarely obtained, although the volume retention of the aerogels remained to be relatively high.

To minimize the crack development in a scale-up production, TMA was employed as a base additive and pore size regulator in the aerogel preparation. It was observed that the addition of small amount of TMA did not significantly affect the volume shrinkage of the aerogel (The volume shrinkage of the aerogels obtained in the presence and absence of TMA were generally around 25 28\%), but resulted in a slightly reduced Young's modulus of the aerogels. Therefore, TMA concentration should be kept at a minimum level.

Fig. 3 (a) and (b) illustrate the MTMS-VTMS based aerogels prepared in the presence and absence of TMA. It is clearly demonstrated that the use of TMA effectively controlled the crack development, enabled a large piece of monolithic aerogel to be successfully prepared at ambient pressure. The SEM image in Fig. 3 (c) (SEM, FEI Phenom) shows a highly porous nature of the aerogel, with a significant proportion of large pores (in micrometers). Further characterisation with the nitrogen adsorption-desorption isotherm is required to reveal the more comprehensive microstructure of the aerogel. This analysis is not included in this paper. However, it is known that MTMS based aerogels generally contain hierarchical multi-level pore structures $[4,5]$.
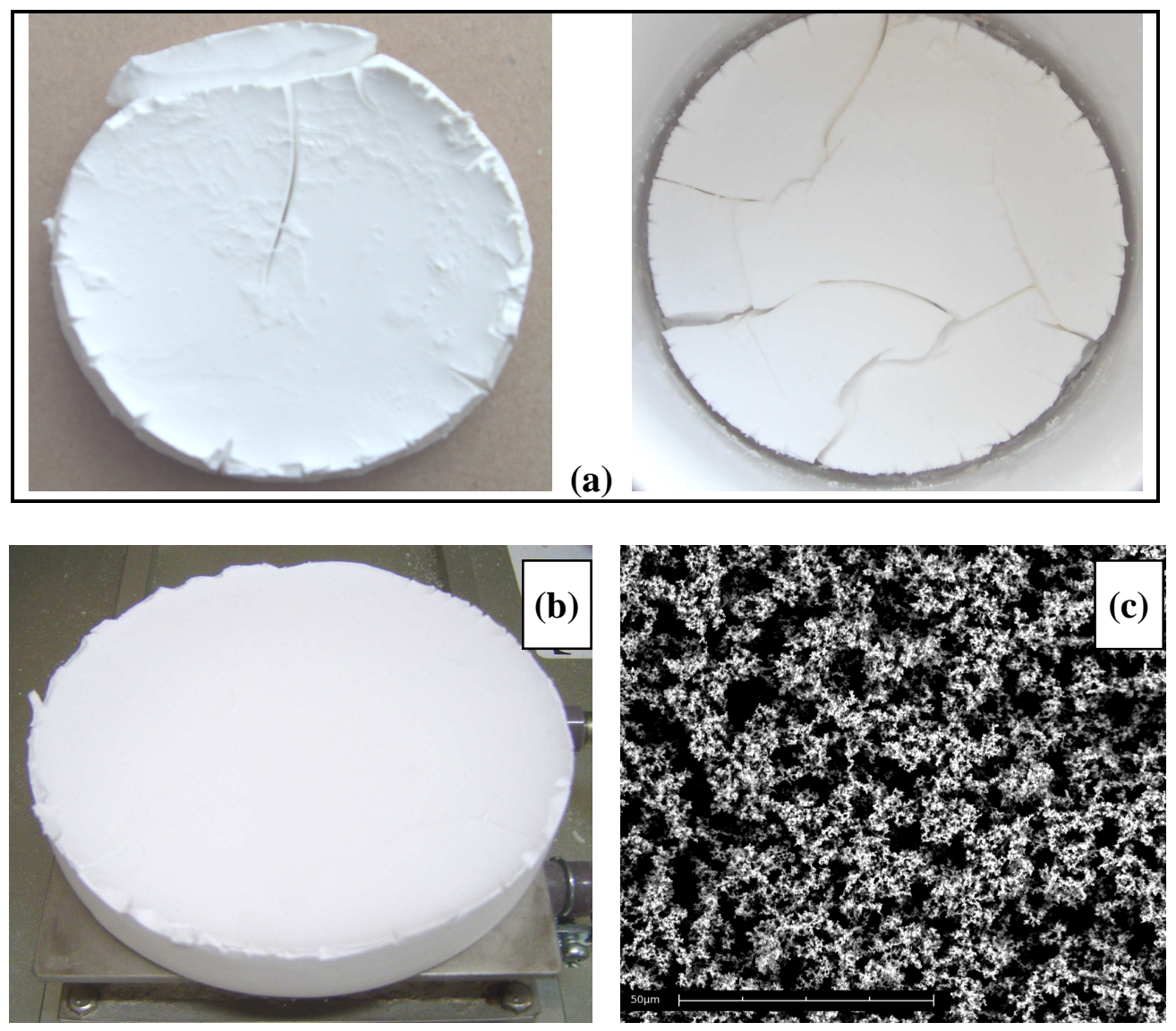

Figure 3. Morphology of the aerogel prepared (a) Aerogels prepared without TMA; (b) An aerogel prepared in the presence of TMA; (c) a SEM image of Sample b 
Thermal Insulation Property and Bulk Density. To evaluate the thermal insulation property of the aerogel produced in this study, a commercially available thermal insulator "Spaceloft ${ }^{\circledR}$ " (from Aspen) was used as a benchmark. Spaceloft ${ }^{\circledR}$ is a flexible aerogel blanket. It combines a silica aerogel with reinforcing fibres to deliver thermal insulation performance, mainly in building applications [8]. For comparison, thermal conductivities of the aerogel prepared in this study and "Spaceloft ${ }^{\circledR}$ " blanket were assessed using the same instrument and testing method. The bulk densities of these samples were also measured. The test results in Fig. 4 show that the aerogel sample prepared in our lab has a thermal conductivity equivalent to the commercial aerogel blanket "Spaceloft®", but its bulk density is significantly lower (i.e. > 30\% lighter) than that of "Spaceloft ${ }^{\circledR} "$ blanket.
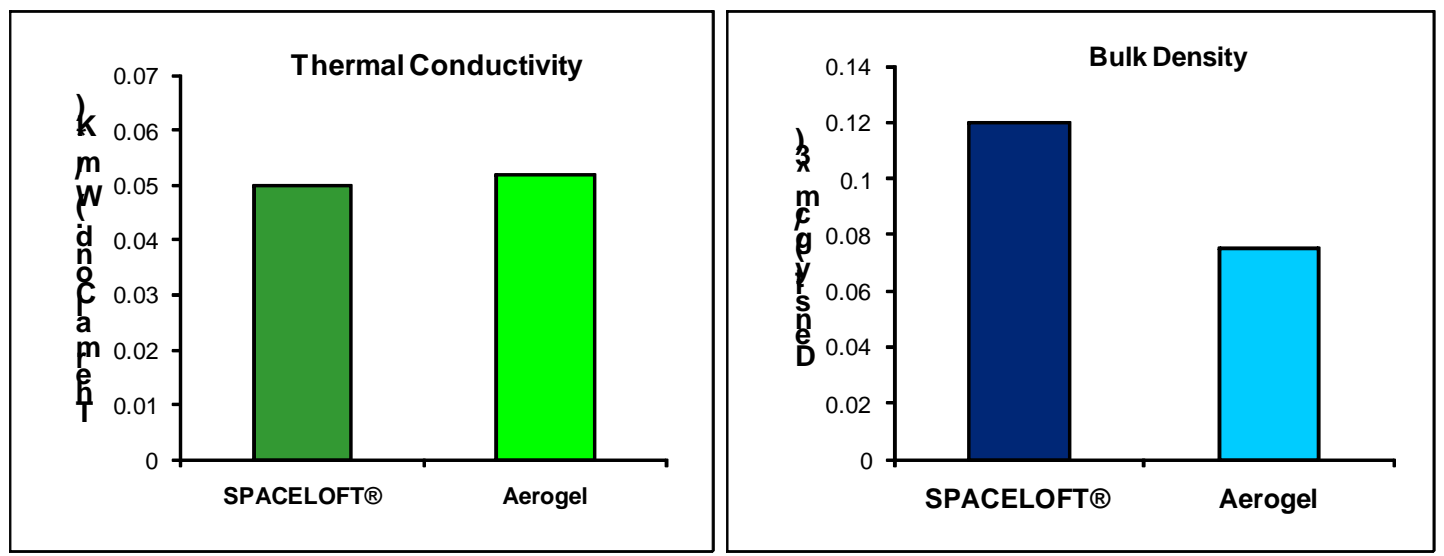

Figure 4. Thermal conductivity and bulk density measurements

Acoustic Insulation Property. The aerogel prepared in this study (with thickness $=1.6 \mathrm{~cm}$ ) was also evaluated for its acoustic insulation performance. For comparison purposes, two other acoustic insulation materials were also tested under the same conditions. These included a commercially used acoustic insulator, an open cell PU foam with a non-woven scrim (Insulator A, thickness $=2.0$ $\mathrm{cm}$ ), and a "high loft" non-woven fibre material (Insulator B, thickness $=4.3 \mathrm{~cm}$ ). The acoustic absorption profiles of the samples are shown in Fig. 5.

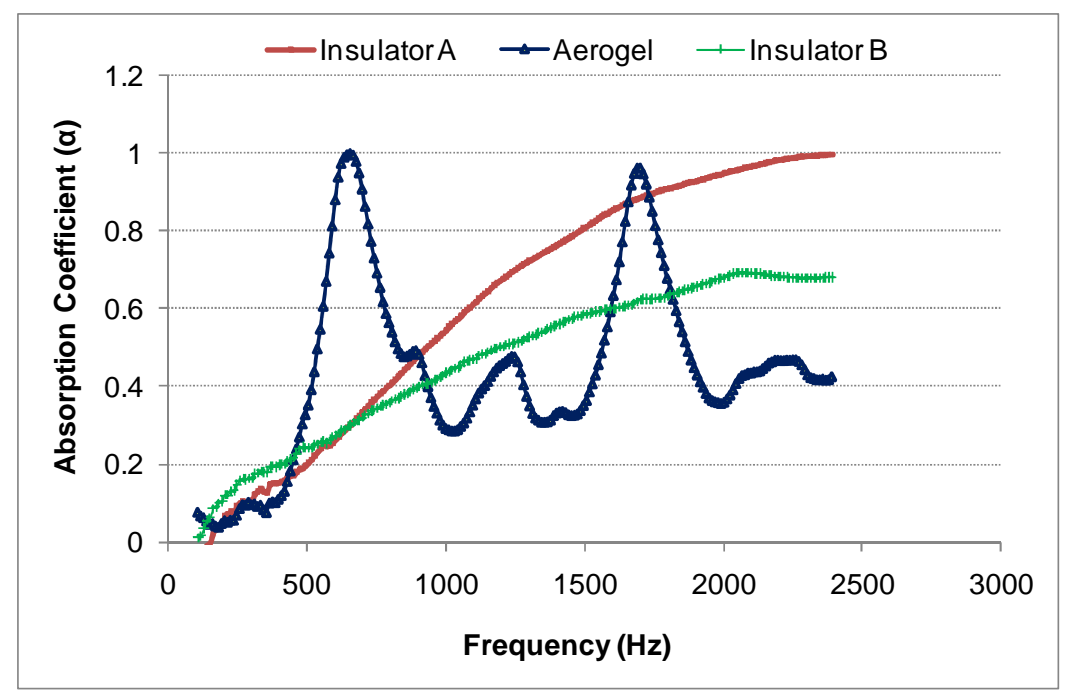

Figure 5. Acoustic property measurements 
It can be seen that Insulators A and B exhibit improved acoustic absorption as frequency increases, and generally low absorption at a low frequency range (Fig. 5), which are porous absorber behaviour basically. The aerogel prepared in this study, on the other hand, exhibits a substantially different profile, with very strong absorption peaks at a low frequency region from 540 to $830 \mathrm{~Hz}$, and at a high frequency region from 1570 to $1860 \mathrm{~Hz}$. The sound absorptions at $610-700 \mathrm{~Hz}$ and $1660-1730 \mathrm{~Hz}$ are over $90 \%$, which is particularly notable. These narrow absorption peaks observed suggest that the aerogel resembles a resonator [9], where the pores act as the resonating cavities. As resonance frequency is inversely proportional to the cavity size [10], the strong absorption peaks observed at both low and high frequency regions also indicate that the aerogel contains both large and small pores. Such a high sound absorption at low frequencies is otherwise very difficult to achieve with normal insulation materials.

Further study on aerogels with different microstructures and aerogel composites are being conducted. The aerogels unique properties will provide opportunities to design and develop new acoustic insulation materials with improved acoustic protection over a broad range of frequencies.

\section{Summary}

An improved process has been developed for the fabrication of large pieces of monolithic aerogels at ambient pressure. The process is based on the use of MTMS and VTMS as precursors and TMA as a base additive to the originally used ammonium hydroxide solution for the alcogel preparation. The aerogels made in this investigation are excellent thermal insulation materials with light weight. The aerogels also exhibit very high absorption in a low sound frequency range, providing opportunities to develop new and improved acoustic insulation materials.

\section{References}

[1] A. Taher, Scientists hail 'frozen smoke' as material that will change world, Sunday Times, 19 August 2007, also on http://www.timesonline.co.uk/tol/news/science/article2284349.ece.

[2] Information on http://www.bccresearch.com/report/aerogels-avm052b.html.

[3] J.Y. Cai, B. Xu, L. Wang, Y. Cao, N. Finn and Z. Cai, Advanced Materials Research Journal, 2011, in press.

[4] B. Xu, J.Y. Cai , N. Finn and Z. Cai, Micropor. Mesopor. Mater. (2011), doi:10.1016/j.micromeso.2011.08.012.

[5] B. Xu, J.Y. Cai, Z. Xie, L. Wang, I. Burgar, N. Finn, Z. Cai and L. Wong, Micropor. Mesopor. Mater. (2011), doi:10.1016/j.micromeso.2011.08.015.

[6] B. Xu, PhD Thesis (2010), Donghua University, Shanghai, China.

[7] A.V. Rao, S.D. Bhagat, H. Hirashima, G.M. Pajonk, J. Colloid Interface Sci. 300 (2006) 279.

[8] Information on http://www.aerogel.com/Aspen_Aerogels_Spaceloft.pdf.

[9] Information on http://www.mixonline.com/online_extras/sound_absorbing_materials/.

[10] S. Sakamoto, M. Hikari and T. Hideki, J. Acoust. Soc. Jpn. (E), 21 (2000) 9-15. 